\section{Sentinellymphknotenbiopsie beim malignen Melanom: ja oder nein?}

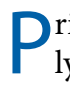
rimär ist die Biopsie der Sentinellymphknoten eine diagnostische Maßnahme, um das Tumorstadium festzulegen, die adjuvante Therapie abzustecken und die Prognose einschätzen zu können. Die diagnostischen Vorteile, so PD Dr. Ulrike Leiter-Stöppke, seien gegen die mit der Biopsie und eventuell nachfolgenden komplettierenden Lymphknotendissektion verbundenen Risiken sorgfältig abzuwägen.

Die Entscheidung für eine Biopsie der Wächterlymphknoten fällt laut aktueller S3-Leitlinie anhand der Tumordicke. Ab einer Tumordicke von $1 \mathrm{~mm}$ steigt der Anteil positiver Sentinellymphknoten. Die Biopsie wird daher ab einer Tumordicke von $1 \mathrm{~mm}$ empfohlen, wenn keine Hinweise auf lokoregionale oder Fernmetastasen vorliegen. Neben der Tumordicke sind Ulzeration, erhöhte Mitoserate und Lymphangiosis Parameter, die die Wahrscheinlichkeit für einen Sentinellymphknotenbefall erhöhen. Die Leitlinie empfiehlt deshalb bei einer Tumordicke von $0,75-1 \mathrm{~mm}$ ebenfalls eine Sentinellymphknotenbiopsie, wenn zusätzliche Risikofaktoren wie Ulzeration, erhöhte Mitoserate oder Lebensalter unter 40 Jahren vorliegen. In der 8. Auflage des Cancer Staging Manual der American Joint Committee of Cancer (AJCC) von 2017 wird die Mitoserate als Staging Kriterium nicht mehr berücksichtigt.

Fällt der Sentinellymphknotenbefund positiv aus, verschlechtert sich die Prognose unabhängig von der Tumordicke. Die Prognose korreliert mit der Tumorlast, wobei noch unklar ist, welche Kriterien als Maß der Tumorlast prognostisch am aussagekräftigsten sind. Relevant für die Prognose sind die Anzahl der positiven Sentinellymphknoten, der maximale Metastasendurchmesser, das Vorliegen einzelner Melanomzellen versus Zellgruppen, die maximale Eindringtiefe in das Lymphknotenparenchym, eine Infiltration der Lymphknotenkapsel oder ein Kapseldurchbruch, die Lage der Tumorzellen im Lymphknoten und eine Lymphangiosis.
Soll nach positivem Wächterlymphknotenbefund eine komplettierende Lymphknotendissektion stattfinden oder nicht? In der multizentrischen randomisierten Phase III-Studie De-COG-SLT, in der nach positiver Sentinellymphknotenbiopsie 240 Patienten einer komplettierenden Lymphknotendissektion zugeführt und 233 Patienten beobachtet worden waren, verbesserten sich durch den Eingriff weder das rezidivfreie, noch das fernmetastasenfreie Überleben oder das Gesamtüberleben. Unterschiede ergaben sich erst, wenn die Größe der Mikrometastasen und die Größe des Tumors berücksichtigt wurden [Leiter U et al. Lancet Oncol 2016; 17: 757-67]. Angesichts der mit dem Eingriff verbundenen schweren Nebenwirkungen sollte eine komplettierende Lymphknotendissektion deshalb nach derzeitigem Stand nur bei Patienten mit Mikrometastasen von über $1 \mathrm{~mm}$ empfohlen werden. Bei einer Metastasengröße von 0,1-1 mm kann der Eingriff angeboten werden, bei Metastasen unter $0,1 \mathrm{~mm}$ oder bei Einzelzellen im Sentinellymphknoten lässt sich gänzlich darauf verzichten. Kathrin von Kieseritzky

S01/04: Melanom - Indikation für SLNB?

\title{
Neue medikamentöse Therapien beim Merkelzellkarzinom
}

$\mathrm{N}$ ur circa 0,4 neue Merkelzellkarzinomerkrankungen pro 100.000 Einwohner werden in Deutschland jedes Jahr diagnostiziert. Doch für die Betroffenen meist ältere immunsupprimierte Personen jenseits des 70. Lebensjahres - endet die Erkrankung oft tödlich. Je nach Größe des Primärtumors überleben lediglich 50-75\% der Patienten die ersten fünf Jahre - liegen bei der Erstdiagnose bereits Metastasen vor, geht das 5-Jahresgesamtüberleben auf 0-18\% zurück. Daran könne die bei Metastasierung bisherige Standardchemotherapie mit Platin und Taxanen nichts ändern, wie Prof. Axel Hauschild, Kiel, ausführte: 50\% Remissionen werden darunter erreicht, jedoch kehrt der Tumor in der Regel nach zwei Monaten zurück, weshalb sich das Gesamtüberleben nicht verbessert.
In einer multizentrischen Phase-IIStudie an 88 Patienten mit chemoresistentem Merkelzellkarzinom im Stadium IV erwies sich eine Therapie mit dem Anti-PD-L1-Antikörper Avelumab (10 $\mathrm{mg} / \mathrm{kg}$ alle zwei Wochen) als effizient: 28 $(31,8 \%)$ von 88 Patienten sprachen auf die Therapie an, darunter acht mit kompletter und 20 mit partieller Remission. Bei 23 von 28 Patienten (82\%) hielt die Remission über längere Zeit an. Das mediane progressionsfreie Überleben betrug 2,8 Monate, das mediane Gesamtüberleben 11,3 Monate [Kaufman HL et al. Lancet Oncol 2016; 17: 1374-85]. Die guten Ergebnisse der Studie führten in den USA zur Zulassung von Avelumab bei Erwachsenen und Kindern ab zwölf Jahren mit metastasiertem Merkelzellkarzinom, unabhängig davon, ob sie bereits eine
Chemotherapie hinter sich haben oder nicht. Für die EU wird die Zulassung laut Hauschild für den Herbst 2017 erwartet.

Ähnlich wirkungsvoll erwies sich der Anti-PD1-Antikörper Pembrolizumab in einer Phase-II-Studie. 26 Patienten mit metastasiertem Merkelzellkarzinom, davon $92 \%$ im Stadium IV, wurden in der Erstlinientherapie mit Pembrolizumab (2 $\mathrm{mg} / \mathrm{kg}$ alle drei Wochen) behandelt. 14 von 25 Patienten sprachen auf die Therapie an, davon vier mit kompletter und zehn mit partieller Remission. Zwölf der 14 Patienten befanden sich nach 33 Wochen Follow-up noch immer in Remission - und dies sogar, wenn die Therapie wegen zu starker Nebenwirkungen (Myokarditis, erhöhte Leberenzyme) schon frühzeitig abgebrochen werden musste [Nghiem PT et al. N Engl J Med 2016; 374 : 2542-52]. Kathrin von Kieseritzky

S11/05: Neoadjuvant - kurativ - palliativ: Was leisten medikamentöse Therapien bei fortgeschrittenen Tumoren? 